\title{
INFLUENCIA DE LAS CARACTERÍSTICAS DEMOGRÁFICAS Y SITUACIONALES EN LA SATISFACCIÓN Y TOMA DE DECISIONES DE LAS ACTIVIDADES TURÍSTICAS A TRAVÉS DEL MOBILE MARKETING
}

\author{
Lázaro Florido-Benítez. \\ Universidad de Málaga
}

\section{RESUMEN}

La democratización del medio digital en las polis turísticas, ha hecho que los humanos se transformen en personas bifuncionales (Humano-smartphone), estos dispositivos han cambiando el paradigma en la toma de decisiones de las actividades turísticas cuando viajamos y, por ende, condicionan al turista su nivel de satisfacción global de su entorno más inmediato. Las factores demográficos y situacionales, son el germen de la adopción de la toma de decisiones de actividades como: búsqueda de información, nuevas experiencias o la compra de productos y servicios a través de los teléfonos inteligentes.

Palabras clave: Mobile marketing; satisfacción; características demográficas y situacionales; transversalidad interaccional digital.

\section{Influence of demographic and situational features in the satisfaction and decisions of} tourism activities through Mobile Marketing

\section{ABSTRACT}

The democratization of digital media in touristic cities has transformed humans into bifunctional people (Human-Smartphone). Mobile devices have caused a paradigm change in how we make decisions about touristic activities when we travel; consequently, this

Recibido: 9 de marzo de 2015

Devuelto para su revisión: 23 de septiembre de 2015

Aceptado: 10 de noviembre de 2015

Facultad de Turismo. Universidad de Málaga.Campus de Teatinos.29071 MÁLAGA(España).E-mail: lfb@uma.es 
conditions the level of global satisfaction a tourist experiences in his or her most immediate environment. Thus, both demographic and situational factors are the seeds of the decisions adopted on various activities such as searching information, new experiences, or the purchasing of products and services on a smartphone.

Keywords: Mobile marketing; satisfaction; demographic and situational characteristics; digital interactional transversality.

\section{INTRODUCCIÓN}

El sector turístico es uno de los entornos más complejo y competitivo que deben sustentar y actualizar la trepidante revolución de las Tecnologías de la Información y Comunicación (TIC). Además, atiende a unas particularidades como: consumidores más informados, expertos y abiertos a los dispositivos móviles, así como adquirir nuevas experiencias por cuenta propia. Los nuevos turistas planifican y estructuran su viaje en función de gustos y prioridades, abandonan las agencias de viajes físicas y reservan sus travesías a través de las agencias virtuales, que le ofrecen unos mejores márgenes económicos, posibilidad de comparar precios y servicios, a la vez que ofrecen una mayor flexibilidad espacio-tiempo y sobre todo mayor diversidad de servicios, es decir, un nuevo perfil de turista, más libre a la hora de seleccionar su viaje y sabiendo lo que quiere, como y cuando. En esta línea tienen que ir todas las estrategias de marketing para atraer clientes interesados y se transformen en clientes reales, para incrementar la tasa de conversión.

La compra y consumo de los productos y servicios turísticos, es una manifestación del comportamiento del turista, que viene determinado por una necesidad, actitud y una motivación emocional que previamente ya ha pensado y adoptado, hasta que la transforma en realidad. Es muy importante la actitud de la persona, ya que ésta la conforma los valores del individuo, es decir, la personas piensan sobre situaciones, productos, marcas, destinos turísticos, gastronomía, etc. en función de los valores que ha adquirido. Un ejemplo de ello puede ser, para la mayoría de la gente, un viaje a Bora Bora y descansar una semana en sus playas paradisíacas es una ilusión, sin embargo, según Sánchez (2014) los chinos no les gusta la playa, a muchos de ellos le resulta incómodo el sol e incluso hay una cierta cultura de mantener la piel lo más blanca posible. Esas actitudes tienen unos impactos en los comportamientos, en las intenciones de compra, en la frecuencia de consumo o en la repetición de la compra. Son dinámicas, y experimentan cambios de forma más rápida que los valores.

Esta última década se ha producido una revolución comercial y tecnológica en el ámbito del mobile marketing, todo ello inmerso en esta nueva era digital. Lo que está perfectamente claro, es que hombres y mujeres han pasado de ser personas monofuncionales a ser sujetos bifuncionales. Manejamos la mayoría de nuestra información dependiendo de un ordenador, tablet, dispositivos móviles, etc. y todo al alcance de la mano (FloridoBenítez, del Alcázar y González, 2014b).

No obstante, el comportamiento del turista también está influenciado por los aspectos demográficos y situacionales, dichas variables hacen que modifiquen el comportamiento, 
así como la actitud en perfiles semejantes. Por ello, individuos que tengan la misma edad, género y clase social, pueden actuar y tomar decisiones totalmente opuestas. Así pues, la satisfacción definida bajo la perspectiva global, es una valoración referida a un conjunto de experiencias vividas con un producto que se basa en la evaluación de la capacidad de la empresa de entregar de una manera continuada los atributos o beneficios que el consumidor busca. Por lo tanto, en el contexto empresarial, la medida de la satisfacción se convierte en un referente fundamental del éxito pasado, actual y futuro de un negocio (Anderson, Fornell y Lehmann, 1994).

Se manifiesta en dicha investigación, que la satisfacción está influenciada por las características del cliente, por el entorno o ambiente donde se consume, por la imagen, la experiencia, seguridad, expectativas, etc. Además, debemos destacar que la satisfacción como constructo multidimensional no obtiene los mismos resultados y esfuerzos por parte de la empresa en un entorno físico que interactivo. En un medio interactivo la inversión por parte del empresario es mayor y la desconfianza del consumidor aumenta, dado que el servicio/producto o información que recibe el cliente es a través de un dispositivo inalámbrico, es decir, lo observa por una pantalla, pero no físicamente como está acostumbrado los clientes.

Wang, Xiang \& Fesenmaier (2012) examinaron los comentarios de aplicaciones de los smartphones de los turistas e identificaron catorce impactos diferentes de uso de los smartphones: resolución de problemas, búsqueda de información, flexibilidad del viaje, emociones, intercambios de experiencias y memorias de almacenamiento. Es importante destacar que estos estudios demostraron que el uso de la tecnología basada en la ubicación juega un papel clave en la adquisición de conocimiento geográfico y el comportamiento, que a su vez, contribuye a los diferentes componentes que afectan a la estructura de la experiencia (Tussyadiah, 2012). Anderson, Klein y Widener (2008) tratan a los clientes como único agente endógeno y evaluador del proceso de creación de valor y demuestran que la incorporación de las características de los clientes, en los modelos que relacionan el concepto de servicio para la satisfacción del cliente genera manifiestamente modelos más ajustados que evidencian las diferencias reales en que los clientes valoran.

Los objetivos nucleares de esta investigación se focalizan en analizar como las características demográficas y situacionales de los turistas influyen en los niveles de satisfacción y toma de decisión de los productos y servicios turísticos a través de la herramienta mobile marketing. En segundo lugar, estudiar qué factores conductuales y tecnológicos tomaremos en cuenta a la hora de articular estrategias de marketing, para reducir la desconfianza inicial del consumidor en la toma de decisión de compra a través del mobile marketing.

Las guías turísticas a través del móvil han suscitado un gran interés en el campo de la investigación durante la última década, resultando numerosas la gran cantidad de aplicaciones móviles basadas en la web del destino. Particular énfasis se ha dado en la personalización de los servicios, generalmente enfocadas a la recomendación del viaje para ayudar a los turistas a seleccionar los lugares que quieren visitar, este sistema cubre un aspecto importante de la personalización del viaje y, por tanto, reduce la carga de información, los comportamientos, las evaluaciones o valoraciones de otros turistas con intereses similares, lo que potencialmente proporciona terreno para la producción cooperativa de mejora de contenido turístico y las recomendaciones de viajes (Gavalas \& Kenteris, 2011). Según 
Hopkins y Turner (2012) la gente compra la mayoría de los productos por la forma en la que eso les hace sentirse, en otras palabras, adquieren productos por motivos emocionales. Finalmente, estos autores nos dan una pruebas más, que el estado emocional condiciona la toma de decisión en ese momento en concreto, por eso, los contenidos en los dispositivos móviles deben ser concretos e intuitivos.

\section{REVISIÓN DE LA LITERATURA}

\subsection{Mobile marketing}

Aunque el concepto de mobile marketing, parece ser una concepción implantada desde hace mucho tiempo, esta percepción es errónea. Su espacio temporal de actuación como nueva actividad del marketing no es superior a una década, debido a que es una modalidad reciente en el marketing y además está inmersa en el sector de las nuevas tecnologías, con lo cual está expuesta a continuos cambios. Esto ha provocado que su definición reaparezca de diferentes formas y de distintas interpretaciones. La revisión de la literatura evidencia un interés muy reciente por el análisis de esta herramienta, dado que para algunos investigadores y asociaciones como Mobile Marketing Association (MMA) difieren a la hora de definir y enfocar dicho concepto, aunque la mayoría de estos autores como (Martín de Bernardo \& Priede, 2007; Hermoso de Mendoza, 2009; Melendo 2010; Kaplan, 2012) conceptualizan el mobile marketing como un instrumento de esta actividad, en la cual pueden desarrollar sus diseños, publicidades y acciones de marketing. En concreto para Kaplan (2012) lo define como: «Cualquier actividad de marketing llevado a cabo a través de una red ubicua a la que los consumidores están constantemente conectados mediante un dispositivo móvil personal».

No obstante, autores como (Vlachos y Vrechopoulos, 2007; MMA, 2009; Shankar y Balasubramanian, 2009) defienden que este instrumento es un canal de comunicación, donde las empresas pueden comunicarse con sus clientes interactivamente, en concreto para Shankar y Balasubramania (2009) es un canal bidireccional o multidireccional de comunicación y promoción de la oferta entre una empresa y sus clientes utilizando un medio móvil, dispositivo o tecnología. Sin embargo, para (Dickinger, Haghiran, Murphy y Scharl, 2004; Norm, 2011) apuestan por esta modalidad del marketing como un canal de ventas donde poder ofrecer productos y servicios a los usuarios.

Basándonos en las conceptualizaciones de esta revisión bibliográfica, nos alineamos en este trabajo de investigación en la definición del mobile marketing de los siguientes autores Florido-Benítez, del Alcázar y González (2014a) como una herramienta de comunicación y/o producto-servicio, que se utiliza en medios inalámbricos interactivos, en la cual se suministra al cliente la información o producto-servicio personalizado, a través del soporte de cualquier dispositivo móvil.

Asimismo, debemos destacar en esta investigación las aplicaciones móviles, que no es otra cosa que un software adaptado a un dispositivo móvil y que se integra como un instrumento más del mobile marketing. Respecto a la definición de la app, los autores exponen dicha conceptualización desde puntos de vistas y enfoques diferentes. Su literatura es escasa debido a que es un concepto inmaduro y que está actualmente en un proceso 
de desarrollo y expansión. Inicialmente comenzaremos por los autores (Scolari, Navarro, Pardo, García y Soriano, 2009; Bellman, Potter, Hassard, Robinson y Varan, 2011) que defienden la aplicación como una herramienta de promoción, donde poder generar notoriedad y una imagen positiva de marca.

Lo que se pretende es tener una repercusión mediática y fomentar un proceso viral interactivo, mediante publicidad, ofertas, descuentos, premios, etc. Autores como (MMA, 2011; García, 2012) justifican la aplicación como un producto y en la cual aporta valor a la gestión, información y resolución al usuario interactiva y automáticamente.

A diferencia de los autores anteriores (Costa, Barragáns y Rey, 2012; The AppDate, 2012) conciben la aplicación como un canal de venta, donde la distribución es la puerta de entrada para dicho canal y le permite vender los productos y servicios de la empresa. Además la empresa tiene la posibilidad de acordar contratos con otras empresas para ofrecer en su aplicación: publicidad, banners, enlaces con páginas de interés, productos y servicios de otras compañías, etc.

Por último, Sanz, Martí y Ruiz (2012) presenta a la aplicación móvil como una herramienta de gestión, donde el usuario tiene la posibilidad de desarrollar determinadas tareas como: búsqueda, información, localización, etc.

Basándonos en la revisión bibliográfica anterior de la app, estableceremos nuestra propia conceptualización de aplicación. Tomaremos una definición más actual, ya que esta herramienta recientemente posee unas dimensiones multifuncionales y se transforma en un soporte para comunicar, impactar, relacionarse, intercambiar, publicitar y comercial desde el punto de vista del marketing. La app al cubrir una necesidad de forma inmediata, independiente y personalizada, es el soporte más directo para articular estrategias de marketing, usando unos criterios de segmentación rigurosos (MMA, 2011). Expuestas las bondades de este software para los dispositivos móviles, nos inclinamos en este trabajo de investigación a redefinir el concepto de app según Florido-Benítez, del Alcázar y González (2014a) es aquel software que se utiliza en un dispositivo móvil como herramienta de comunicación, gestión, venta de servicios-productos orientados a proporcionar al usuario las necesidades que demande de forma automática e interactiva.

\subsection{Revisión de la conceptualización de satisfacción}

En cuanto al enfoque del ámbito de la referencia, la conceptualización de la satisfacción puede abordarse desde una de estas dos aproximaciones: el enfoque basado en una transacción específica y el enfoque de satisfacción global o acumulada (Jones \& Such, 2000; Yang \& Peterson, 2004). El enfoque basado en una transacción específica define la satisfacción como la respuesta del consumidor a la transacción más reciente con la organización (Oliver, 1993), por lo que se verá influida por las variables situacionales presentes en ese momento. La satisfacción es un juicio evaluativo post-elección y/o una respuesta emocional del consumidor ante una determinada compra o una ocasión de consumo (Spreng, MacKenzie \& Olshavsky, 1996; Giese \& Cote, 2000). Así queda reflejado claramente en la definición de Giese \& Cote (2000) cuando indican que la satisfacción es una respuesta que se da en un momento temporal específico, de duración limitada y referido a determinados aspectos de la compra y/o consumo de un producto. De ahí que la mayoría 
de las investigaciones que analizan el proceso de formación de la satisfacción adopten la perspectiva de la transacción específica (Vanhamme \& Snelders, 2001; Helgesen, 2007).

Asimismo, la satisfacción global o acumulada considera que el juicio emitido por el consumidor es el resultado de una acumulación de experiencias, incluyendo tanto la satisfacción asociada con productos específicos como la relativa a diferentes facetas de la empresa (Cronin y Taylor, 1992; Homburg y Giering, 2001). De igual modo, Woodruff, Schumann y Gardial (1993) y Woodruff y Gardial (1996) entienden que la satisfacción es un sentimiento que procede de una experiencia inmediata o un conjunto de experiencias. Según Cook, Goh y Chung (1999) observaron que la mayoría de las tipologías de servicios se centran exclusivamente sobre las características discriminantes del concepto de servicio, ignorando las características de los clientes y tratando implícitamente a todos los usuarios por igual. Por lo tanto, es evidente que todos los clientes no son iguales y ni actúan de manera similar en entornos interactivos, los desarrolladores de la apps en los aeropuertos tendrán que estructurar sus contenidos en función de sus pasajeros objetivos.

En el contexto de los servicios, también Cronin y Taylor (1994) sugieren que la satisfacción es una evaluación acumulativa y, por tanto, representa más un juicio global que una medida de una transacción específica. Así pues, la satisfacción definida bajo la perspectiva global, es una valoración referida a un conjunto de experiencias vividas con un producto que se basa en la evaluación de la capacidad de la empresa de entregar de una manera continuada los atributos o beneficios que el consumidor busca.

En este trabajo de investigación asumimos que el concepto de satisfacción es bastante amplio y que en este intervienen multitud de variables como hemos podido observar en la literatura referenciada de dicha conceptualización. Por ello, hemos visto necesario establecer nuestra propia definición de satisfacción, con la finalidad de exponer y clarificar nuestros planteamientos (Florido-Benítez, del Alcázar y González, 2014b) la satisfacción es la evaluación global o acumulada de una experiencia de consumo de un producto o servicio, donde intervienen componentes cognitivos, afectivos, situacionales (experiencia, clase de servicio, etc.) y personales como (sexo, edad, ingresos, etc.).

Traslademos estas variables analizadas a la satisfacción de un turista que está utilizando la herramienta mobile marketing. Variables como: imagen, expectativas, calidad del servicio/producto, disponibilidad, ubicuidad, estado cognitivo y afectivo, experiencia, seguridad, componentes personales, valor percibido, dado que estamos utilizando un dispositivo móvil y que interactuamos en línea con la app de un destino turístico. Estos atributos influirán más o menos en la evaluación de la satisfacción y determinarán hacia que lado de la balanza se inclinará el cliente, entre la lealtad o la queja.

A continuación exponemos en la siguiente figura, la adopción de estos dos factores (nuevas tecnologías y características de comportamiento del usuario), para reducir las desventajas y desconfianza del cliente, con la finalidad de aumentar los niveles de satisfacción global del usuario y facilitar la toma de decisión de compra de un servicio.

Evidentemente, estos factores como (incertidumbre, la desconfianza inicial, la forma de pago, mejora de la conexión a internet, déficit de rapidez en la navegación, seguridad, conveniencia, cultura, información de la transacción del pago, la incompatibilidad de ciertas apps a determinados dispositivos móviles, etc.), si no se gestionan bien por parte de las empresas, se transforman en desventajas para el cliente y al final promedian negativamente 


\section{Figura 1 \\ ADOPCIÓN DE FACTORES (TECNOLÓGICOS Y CONDUCTUALES) PARA REDUCIR LA DESCONFIANZA DEL USUARIO EN LA TOMA DECISIÓN}

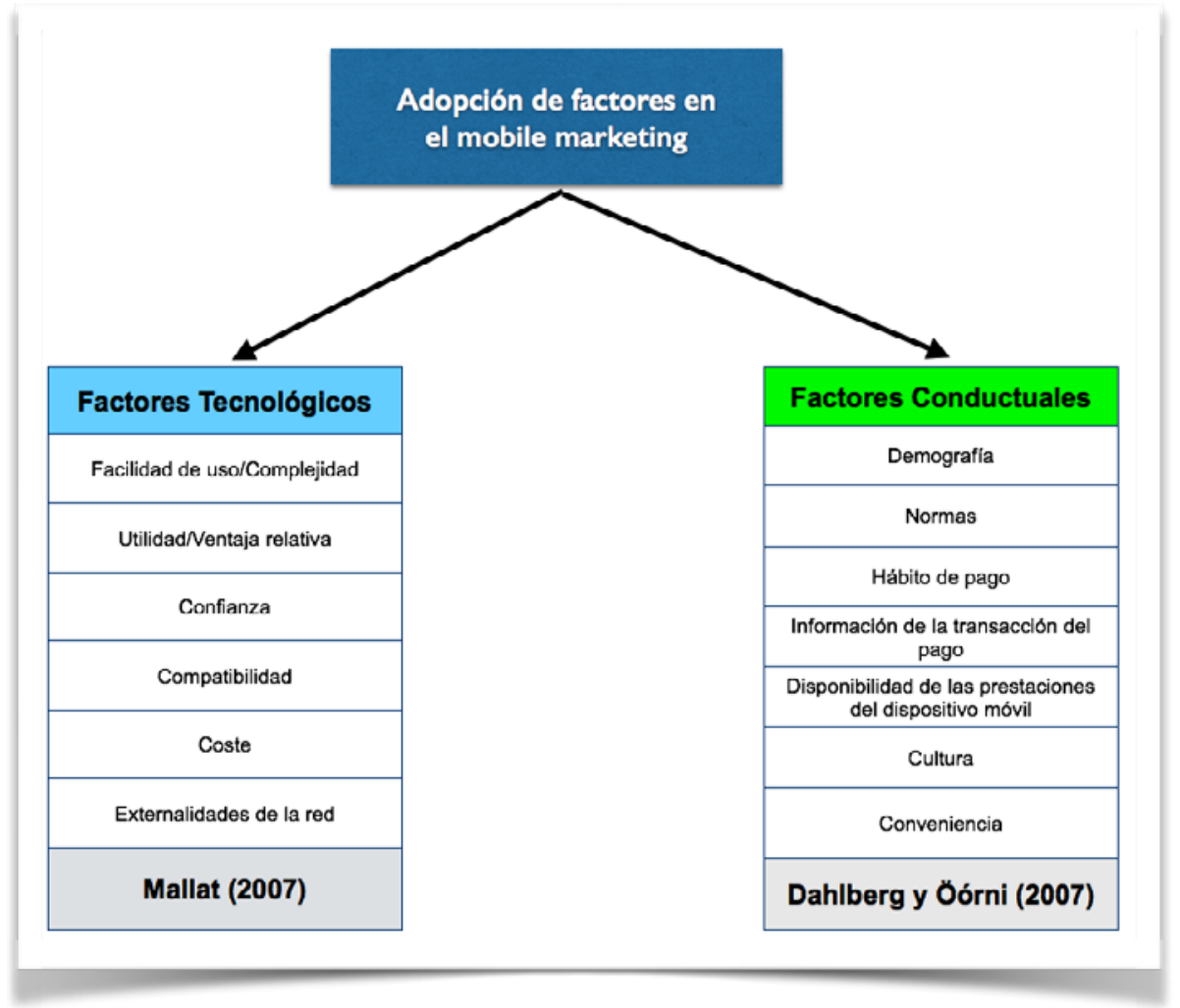

Fuente: Mallat (2007) y Dahlberg \& Öörni (2007).

en la toma de decisión del turista y la satisfacción global de su experiencia. Potenciar sus ventajas y reducir o eliminar sus limitaciones depende en buena parte del trabajo de los creativos, independientemente de cómo se les califique: publicitarios, de marketing, de comunicación (Baños, 2007). El objetivo es lograr que se pueda transmitir la idea a través de una pantalla muy reducida, y para lograrlo, el equipo creativo tendrá que hacer un esfuerzo añadido para crear un mensaje que transmita el concepto que se comunica a través de otros medios, pero adaptados a las características del móvil, especialmente el tamaño de la pantalla y el momento de la recepción.

\subsection{Características demográficas}

Las variables demográficas hacen referencia a los atributos biológicos del individuo, a su situación familiar y a su localización geográfica. Las más importantes son: edad, 
sexo, estado civil, posición familiar, residencia, etc. Respecto a las variables socioeconómicas, hacen referencia a situaciones o estados alcanzados y conocimientos adquiridos: ocupación, profesión o actividad desempeñada, ingresos periódicos obtenidos, patrimonio acumulado o nivel de estudios alcanzado. El conocimiento sobre los dos tipos de características, demográficas y situacionales, es útil e importante para la creación de segmentos de clientes para campañas de marketing (Anderson et al., 2008). A continuación, analizaremos los efectos moderadores en las características demográficas sobre la satisfacción y toma de decisión del turista.

\subsubsection{Género}

(Bryant y Cha, 1996; Mittal y Kamakura, 2001) revelan en sus trabajos de investigación, que las mujeres reportan mayor satisfacción global que los hombres. Una explicación de este resultado es que las mujeres pueden ser mejores compradoras experimentadas y tener mayor habilidad para hacer comparaciones de las particularidades del producto o servicio. La experiencia les permite identificar los mejores elementos de sus necesidades personales y esto les conduce a una mayor satisfacción global que los hombres (Bryant y Cha, 1996). Mittal y Kamakura (2001) sugieren que las mujeres son menos propensas a decir la verdad, acerca de las experiencias negativas que los hombres. Se identifican diferencias en las ponderaciones relativas, que los hombres y las mujeres asignan a los componentes del concepto de servicios que a menudo se atribuyen a las diferencias en el estilo de liderazgo (Eagly, Makhijani y Klonsky 1992) y al estilo del procesamiento de la información. Los resultados sugieren que las mujeres se centran más en los componentes interpersonales de la interacción del servicio (Iacobucci y Ostrom, 1993). De hecho según Zeithaml (1985) las investigaciones han demostrado que las compras de las mujeres están más influenciadas en gran medida por sus evaluaciones de interacciones personales y las consultas al personal de ventas (Gilbert y Warren, 1995), más que las compras de los hombres. Para Anderson et al., (2008) en promedio, las mujeres están más satisfechas que los hombres, la composición del modelo de satisfacción del cliente difiere entre hombres y mujeres y en particular que los elementos de interacción influyen en la satisfacción global más en las mujeres que los hombres. El efecto moderador género en los medios de comunicación interactivos según Okazaki y Hirose (2009) exponen que la mujeres están más predispuestas a los efectos de los medios de comunicación y su correspondiente desplazamiento que los hombres, además, las mujeres tienen una mayor percepción de satisfacción, actitud y uso habitual de internet móvil que los hombres.

\subsubsection{Edad}

Resulta evidente que la edad del cliente va influir en los procesos cognitivos y procedimentales, así como los efectos moderadores en la satisfacción global. Una persona joven codifica e interioriza con mayor rapidez la información que una persona mayor. Las personas mayores, por término medio, son más lenta en la codificación de nueva información y recuperación de la información almacenada en la memoria, reduciendo 
así la capacidad de procesamiento de la información (John y Cole, 1986). Aunque ellos pueden tener pericia desde la experiencia, los beneficios de ésta pericia no son tan fácilmente accesible. Mittal y Kamakura (2001) explican que las personas mayores pueden tener bajos los «umbrales de satisfacción aceptable», porque la búsqueda de información para un nuevo proveedor es más costoso. Sin embargo, algunas investigaciones sugieren que las diferencias relacionadas con la edad en las evaluaciones de productos y servicios pueden ser debido a diferentes expectativas impulsadas por las diferencias en la sociedad y cultura en el nacimiento y la madurez (Bryant y Cha, 1996). En general, las investigaciones anteriores han demostrado que las personas mayores están más satisfechos que las personas jóvenes (Bryant y Cha, 1996; Mittal y Kamakuara, 2001), porque reducen la búsqueda de información y realizan sus evaluaciones en un grupo limitado de alternativas que les son familiares (Janis y Mann, 1997; Smith y Moschis, 1990). Según Liébana-Cabanillas, Sánchez-Férnandez y Muñoz-Leiva (2014) el efecto moderador edad de los usuarios, las empresas tienen que definir estrategias adaptadas a los patrones de influencia, produciendo propuestas de valor diferenciadas, es decir, segmentar su mercado en función de su público objetivo para satisfacer las necesidades de cada uno.

\subsubsection{Ingresos}

A menudo los ingresos se utiliza como sustituto de la educación y por lo tanto, como un indicador potencial del procesamiento de la información interna (Homburg y Giering, 2001). Anteriores investigaciones han estudiado los efectos directos de los ingresos en la satisfacción. Bryant y Cha (1996) detectaron que la satisfacción disminuye a medida que aumenta el ingreso. Aunque la calidad aumente en los productos más caros, estos son comprados y las expectativas también aumentan. Mittal y Kamakura (2001) proponen que los que tenían niveles más bajos de educación, al igual que las personas de edad, poseen niveles más bajos de satisfacción porque la búsqueda de alternativas es más costosa. Aunque los efectos de la interacción de los ingresos y la ponderación de los atributos sobre la satisfacción no se han estudiado antes, podemos predecir, un resultado de un procesamiento más eficiente de la información y una mayor compresión de la capacidad de responsabilidad (Folkes, 1988), los consumidores con mayores niveles de ingresos tienen mayor incidencia en los componentes del servicio. Anderson et al. (2008) exponen que las personas con mayores ingresos ponen menos énfasis en los componentes básicos y al contrario en los componentes periféricos (interaccional y físico), además, a más expectativas la satisfacción va disminuyendo a medida que aumenta los ingresos.

\subsection{Características situacionales}

Respecto a las características situacionales, la experiencia unida al tipo de servicio o producto seleccionado, inicia el proceso de la transversalidad interaccional digital que condicionará el grado de satisfacción del pasajero. En el caso específico del comercio vía móvil, un novedoso canal de compraventa sin explorar para muchos clientes, la 
satisfacción es esencial para el desarrollo y el mantenimiento de la relación, habida cuenta que las primeras experiencias comerciales pueden marcar el éxito o fracaso de este canal para las empresas. Es por ello que múltiples estudios resaltan la importancia de esta variable relacional (Lin y Wang, 2006; Choi, Seol, Lee, Cho y Park, 2008, Lim y Kumar, 2008; Kuo, Wu y Deng, 2009; Akroush, Al-Mohammad, Zuriekat y Algul-Lail, 2011; San Martín y López, 2012).

Estudios previos demuestran que tanto básicos como atributos periféricos se asocian positivamente con la calidad global del servicio y la satisfacción del cliente (Iacobucci y Ostrom, 1993; Chase y Stewart, 1994; McDougall y Levesque, 2000; Butcher 2005). Es por ello que la satisfacción global es un concepto holístico donde intervienen multitud de variables y en el cual cada variable tiene su cuota de incidencia en la satisfacción acumulada del consumidor. Cooil et al. (2007) señalan que las características de los clientes incluyen las características demográficas; situacionales y estado. Es importante investigar los efectos moderadores de ambos tipos y de la situación del cliente, ya que a priori la literatura ha demostrado que ambos son influyentes en la satisfacción del cliente. Las variables demográficas, como la edad, sexo y el nivel socioeconómico, son las propias del cliente. Mientras las características situacionales tales como la experiencia, tipo del servicio y transversalidad son específicas del contexto.

Wangenheim (2003) aportó un ejemplo en una investigación realizada de los efectos moderadores en las características situacionales. En dicho estudio demostró como cuatro características situacionales, incertidumbre de compra/cambio de precio/duración de la relación con el cliente y la importancia de la adquisición moderaban la relación entre la satisfacción del cliente y la lealtad entre oferente-demandante.

\subsubsection{Experiencia}

La experiencia ha sido ampliamente estudiado en el contexto de la búsqueda de información y procesamiento (Alba y Hutchinson, 1987), hay una evidente relación de sus efectos sobre las evaluaciones del servicio. Dado que los repetidos encuentros con un proveedor de servicios son impulsados en gran medida por la autoselección de los leales clientes de un producto, podríamos esperar que la satisfacción global es mayor para los expertos que los noveles (Johnson y Fornell, 1991). Sin embargo, los investigadores han presentado teorías opuestas, como Reinartz y Kumar (2002), quienes argumentan que los clientes leales son más exigentes, como resultado de sus expectativas más altas. Del mismo modo, Zeithaml y Bitner (2002) sugieren que una mayor variedad de la experiencia pasada, puede incrementar el nivel individual de la persona del servicio. Se detecta en dichas investigaciones, que existe relación sobre el efecto de la experiencia en el nivel de satisfacción global, pero no el grado de satisfacción entre expertos y novatos, entre persona joven y persona mayor o entre mujer y hombre. Observamos que los turistas expertos que tienen mayor experiencia a nivel de interacción con el móvil e información de productos y servicios, son más participativos a la hora de comprar que aquellos que no poseen un smartphone, por lo tanto, las expectativas de los más experimentados cada vez son más altas. 


\subsubsection{Clase de servicios}

La clase de servicio puede afectar diferentemente a las expectativas, así como las percepciones del rendimiento si el servicio es visto principalmente distinto entre el pasajero que posee un móvil y tiene más información, a aquel que no posee teléfono móvil o no utiliza la app que el destino turístico ofrece. Por lo tanto, consideramos que es una característica situacional y usada por Cooil et al. (2007). Debido a las expectativas de información del servicio a través del móvil, serán más altas en aquellos clientes que utilizan el smartphone como herramienta de comunicación, que los usuarios que no hacen uso de su dispositivo móvil, la satisfacción es a menudo menor que lo que podría preverse sobre la esencia de los servicios prestados por sí solo (Bryant y Cha, 1996). Los pasajeros que usan la herramienta mobile marketing ponen menos énfasis en los atributos básicos y se inclinan más por los atributos periféricos como la interacción y elementos físicos. Anderson et al. (2008) propone que al tener unas expectativas más altas, la satisfacción va disminuyendo a medida que aumenta la clase de servicio.

Al final sólo estamos ofreciendo productos y servicios calibrados a priori a un segmento de nuestro público objetivo que ya teníamos prederteminado en nuestro plan de marketing. Sasser, Olsen y Wyckoff (1978) acuñaron el término «concepto de servicio» para describir el conjunto de elementos envasados para la venta al cliente. Las operaciones de servicio y estudios de marketing de servicios, postulan dos tipos fundamentales de atributos de servicios: los atributos básicos «los que se entrega» y los atributos periféricos «como se entrega» (Iacobucci y Ostrom, 1993; Chase y Stewart, 1994; McDougall y Levesque, 2000). Iacobucci y Ostrom (1993) definen los atributos básicos como el núcleo de un servicio que es parte de un servicio que nosotros pensamos cuando nombramos el servicio, es decir, si vamos a una cafetería de un centro comercial y solicitamos un café y una tostada, este es el atributo básico y el atributo periférico, es como lo sirven, el ambiente de la cafetería, la atención del camarero, la calidad del producto, la imagen, información, etc.

Los atributos periféricos se dividen en físico, interacción y otras particularidades (Bitner, 1990; Chase y Stewart, 1994). Los atributos físicos incluyen componentes ambientales, mecánicos, e intangibles de la prestación de servicios. Los atributos de interacción incluyen todos los encuentros interpersonales implicados en la prestación de servicios.

\subsubsection{Transversalidad interaccional digital}

Este concepto, aparece como una estrategia fundamental de actuación para mejorar las respuestas y demandas de oferentes-demandantes. Bigné y Andreu (2005), defienden que la interacción y participación en los encuentros del servicio repercuten en la satisfacción. En las características situacionales las empresas tienen una mayor actuación para favorecer e incrementar los niveles de satisfacción global. El cliente percibe cuando utiliza su smartphone que hay una continuidad y sintonía entre la clase de servicio seleccionado, la experiencia en la que está inmersa en ese momento y la transversalidad interaccional digital de los contenidos, información y productos o servicios que consume. "Transvesali- 
dad interaccional digital" es un instrumento organizativo de las empresas, que pretenden desarrollar y monitorizar las estrategias y herramientas que permiten adaptarse mejor a las exigencias del cliente. La transversalidad «tiene como finalidad promover una mejora de la calidad de vida para todos" (Palos, 1988).

La transversalidad interaccional digital, se inicia en el momento que el cliente comienza su proceso de experiencia en buscar la información del vuelo, reserva el billete de avión (producto), demanda una serie de productos y servicios personalizados y las empresas se los proporcionan, así hasta llegar a su destino o finalizar todo el proceso (vía dispositivo móvil) de compra-venta entre oferente y demandante.

Figura 2

DIMENSIÓN DE LA TRANSVERSALIDAD

INTERACCIONAL DIGITAL CON EL MOBILE MARKETING

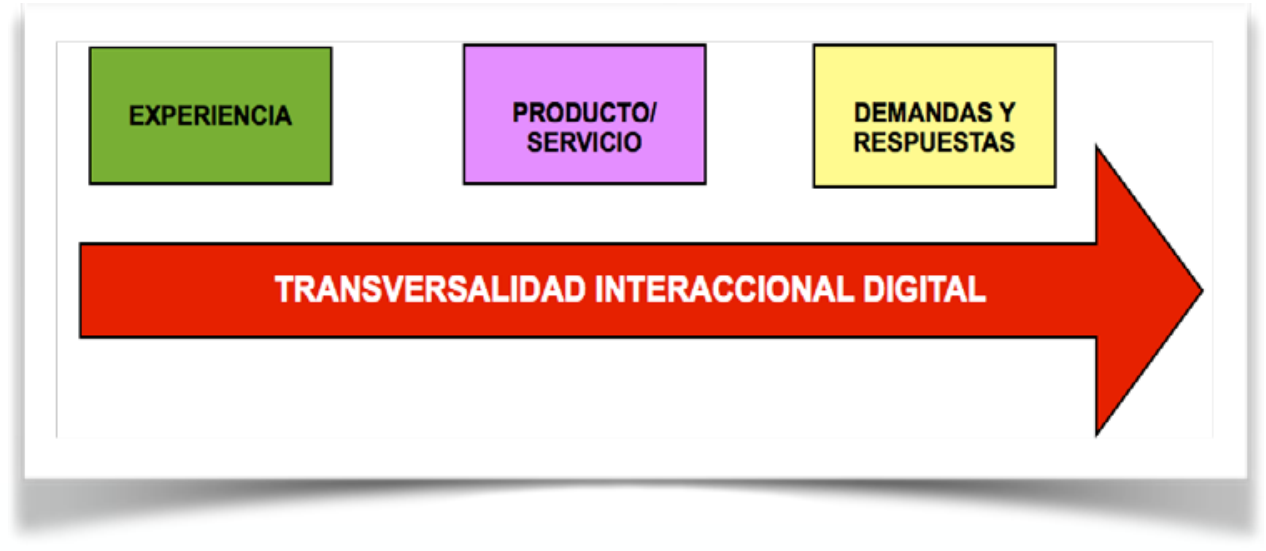

Fuente: elaboración propia.

Evidentemente para que se den estos requisitos, tiene que existir una relación de interoperabilidad entre todos los agentes implicados. Si esta transversalidad se proporciona al consumidor de una manera estructurada, ordenada y satisfaciendo las necesidades del cliente, seguramente favorecerá la toma de decisión del turista e incrementará los niveles de satisfacción.

Proporcionar esta tecnología de información en su smartphone al turista en tiempo real, para mejorar su experiencia y conseguir mayor eficiencia en sus actividades, es bastante complicado. Muchas de las apps móviles necesarias para brindar estos servicios dependen en gran medida de la capacidad de la industria para compartir datos en el momento adecuado, información que probablemente provenga de múltiples fuentes. Por ejemplo, casi dos tercios de las compañías aéreas tienen previsto informar a los pasajeros del estado de su equipaje facturado en tiempo real a través de sus móviles. Pero para proporcionar este servicio también necesitarán el acceso en tiempo real a los datos de todos los involucrados en los procesos de facturación de equipajes que 
pueden incluir el handling en tierra, el aeropuerto e incluso otras compañías aéreas, si el equipaje y el pasajero están siendo transferidos a otro vuelo (Ramón, 2014).

No obstante, en el conjunto de esta actividad mercantil hay un proceso pedagógico, donde debemos entender cómo funciona la interacción entre cliente-empresa, por no decir, el aprendizaje y perfeccionamiento de los turistas con la herramienta marketing en la transformación de un deseo o necesidad hasta que adquiere el producto o servicio, [...] el participante de estas actividades no se transforma en un espectador sino más bien en el protagonista de su propia educación (Castillo, 2010). Nos centraremos en el contenido nuclear de este trabajo, como afecta las características demográficas y situacionales de los turistas a través herramienta mobile marketing y los componentes del servicio (básicos y periféricos), en el resultado de la evaluación de la satisfacción global y mejora de toma de decisiones.

El marketing móvil es interactivo, ya que permite el diálogo entre emisor y receptor. La bidireccionalidad de este medio permite que haya diálogo entre ambos, mejorando el intercambio de información y su relación. Esto es muy ventajoso para los dos. El anunciante, al tener acceso a los gustos, preferencias o necesidades de su público objetivo, tiene una vía directa de contacto con el cliente, que le puede servir, entre otros, como servicio postventa, información al consumidor y ofertas o promociones (Melendo, 2012).

En la siguiente figura 3, se observa que la satisfacción global del pasajero con la herramienta mobile marketing, está condicionada por los efectos moderadores de las características del pasajero. Hui, Wan y Ho (2007), investigaron el impacto de las características demográficas (género, estado civil, ingresos, ocupación y nacionalidad) y detectaron que diferentes aspectos de los servicios ofrecidos daban lugar a diferencias en la satisfacción, recomendación y repetición. Los efectos moderadores de las características demográficas, tienden a generar comunicaciones boca-oído positivas y una mayor intención de repetir la visita al establecimiento (Gil, Ruiz, Berenguer y Corraliza, 2012). Lubbe (2007) defiende que las características demográficas están notablemente relacionadas con la satisfacción.

Asimismo, en la evaluación del cliente con el mobile marketing contemplamos los tres elementos principales, que se ofrezca una buena conexión wifi al usuario, la percepción de ubicuidad del pasajero, el mobile marketing logra la ubicuidad en sus acciones cuando más allá de localizar al individuo geográficamente, se logra localizar al individuo como tal, en función de sus gustos, preferencias, demandas o características y la evaluación de optimización del tiempo por parte del turista, gracias a la instantaneidad del medio, la personalización del mensaje, la cercanía del turista en el momento de recepción de la información o momento de compra y la posibilidad de unir en un mismo canal el medio, mensaje y canal de respuesta.

En la evaluación-constatación del cliente con los elementos periféricos del servicio como: información en tiempo real, señalización digitalizada, accesibilidad orientada, ofertas y descuentos en el dispositivo móvil y los servicios ofrecidos de la aplicación móvil del destino, el pasajero tendrá que evaluar las ventajas o desventajas de esta herramienta y constatar su veracidad, si quiere consumir el producto y servicio o recibir la información demandada. 


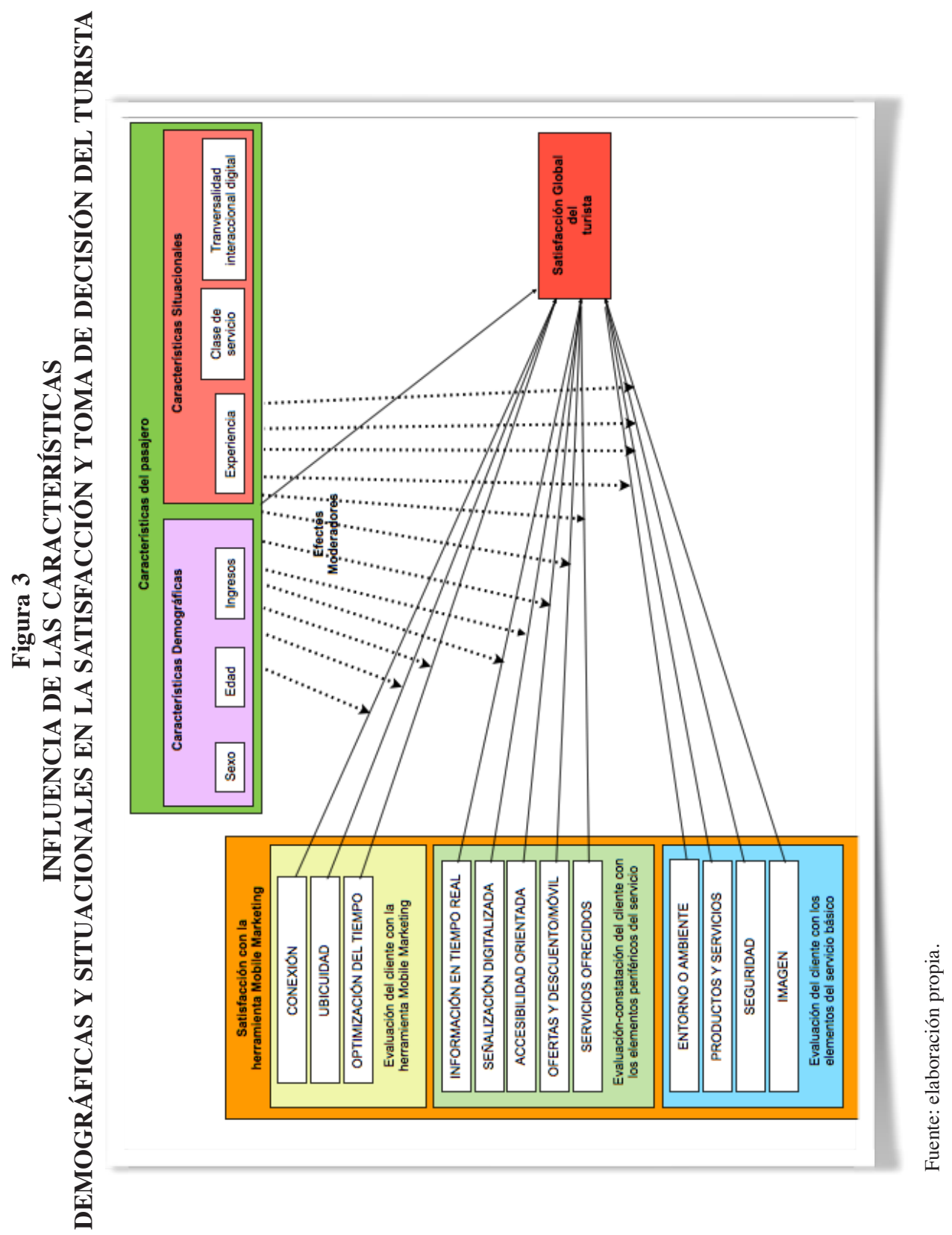




\section{METODOLOGÍA}

La metodología desarrollada en este trabajo ha sido de carácter cualitativo mediante la estrategia denominada «no interactiva analítica documental» (Cohen \& Manion, 2002; McMillan \& Schumacher, 2005). Esta estrategia de investigación consiste en acudir a archivos de datos y fuentes bibliográficas en busca de la información que sobre nuestro problema de estudio han reunido otros autores, instituciones y organismos. Inicialmente, hemos visto necesario exponer las oportunidades que ofrece el mobile marketing tanto a destinos como a turistas. Seguidamente, estudiamos la revisión de la literatura el mobile marketing, satisfacción, las características demográficas y situacionales, así como la variable transversalidad internacional digital. No obstante, es significativo, exponer y analizar la relación de los nexos turista-smartphone, para examinar el valor que se puede entregar a los usuarios a través de la utilización de los servicios del móvil. Según Al-Debei \& Al- Lozi (2014), postulan que las percepciones de los usuarios en relación con el valor que pueden obtener al utilizar los servicios móviles, se ve directamente afectado por las influencias tecnológicas, sociales y de información.

En otras palabras, analizamos la interacción en tiempo real y la conectividad global de Internet del viajero con los dispositivos móviles, evaluando la incidencia de las particularidades del individuo en su toma de decisiones y como promedian positiva o negativamente en la satisfacción. En el marco del marketing-mix, Internet se ha consolidado como una herramienta esencial, transformando todo el tejido empresarial turístico, con el fin de crear nuevas experiencias al turista y teniendo a los dispositivos móviles como rosa de los vientos, para la gestión y planificación de sus viajes. Los destinos turísticos, deberían posicionar sus productos y servicios en el mobile marketing, según Song (2014) de modo que puedan proporcionar a sus potenciales usuarios experiencias positivas.

\section{RESULTADOS}

Atendiendo a la revisión bibliográfica de esta investigación, se detecta que para favorecer la toma decisión de los turista a través del mobile marketing, tendremos que tomar en cuenta la adopción de las nuevas tecnologías y las características de comportamiento del cliente o usuario. Nos gustaría señalar, que debemos tener en cuenta la palabra "adoptar" y que es definida por la Real Academia Española (RAE): «Recibir, haciéndolos propios, pareceres, métodos, doctrinas, ideologías, modas, etc. que han sido creados por otras personas" otra definición de la RAE es: "Tomar resoluciones o acuerdos con previo examen o deliberación". Estas dos definiciones van de la mano en la adopción de factores tecnológicos y conductuales por parte del cliente, dado que este recibe información y gestiona procesos interactivos exógenos a través del dispositivo móvil, en los que tiene que tomar resoluciones con previa deliberación, es decir, el cliente inicialmente no acepta, el cliente adopta e interioriza informaciones y procesos externos, previa deliberación de sus características personales, situacionales, etc. y después toma las decisiones. Según Jinzhu (2014) entender la adopción de la innovación móvil, es investigar las decisiones de los consumidores a aceptar productos o servicios a través de la tecnología móvil a nivel individual. 
Figura 4

TIEMPO QUE PASAN LOS ESTADOUNIDENSES

EN EL USO DE LOS DISPOSITIVOS MÓVILES

\section{TIEMPO POR DÍA QUE PASAN LOS CONSUMIDORES ESTADOUNIDENSES EN EL USO DE LOS DISPOSITIVOS MÓVILES}

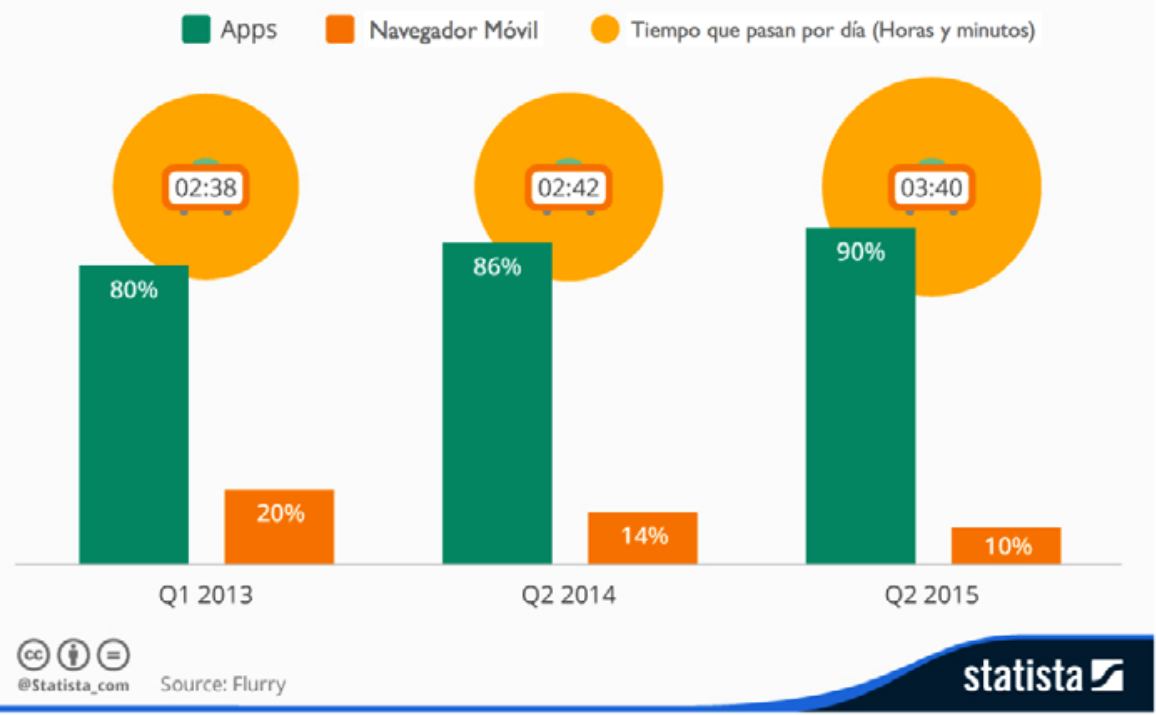

Fuente: www.statista.com y http://flurrymobile.tumblr.com/post/127638842745/seven-years-into-the-mobilerevolution-content-is

Está bien documentado que las características del cliente como por ejemplo: sexo, edad, ingresos, tienen un efecto importante en el nivel de satisfacción del cliente (Johnson y Fornell, 1991; Bryant y Cha, 1996; Danaher 1998; Mittal y Kamakura, 2001). No obstante, los estudios muestran que las características de los clientes moderan los resultados de la satisfacción de este, incluyendo las intenciones de recompra (Cooil, Timothy, Keiningham y Hsu, 2007). Las características de los clientes explican las diferencias en la composición de la satisfacción global (Anderson, Klein y Widenar, 2008). Además, estos autores defienden que la gestión de la satisfacción del cliente requiere conocimiento de los elementos del concepto de servicio y de las características de este para proporcionar una visión completa del proceso de co-creación que genera la satisfacción del cliente.

En nuestro caso de estudio, es muy importante valorar la relevancia de los dispositivos móviles en el día a día de las personas. La consultora Flurry Insights (2015) presentó un informe donde se mostraba que los estadounidenses pasaban un promedio de 3 horas y 40 minutos al día en el uso de los dispositivos móviles, en los trimestres que a continua- 
ción mostramos. El uso de las aplicaciones móviles han ido aumentando, mientras que la utilización de los navegadores móviles han decrecido en un 50\% del primer trimestre de 2013 hasta el segundo trimestre del 2015.

Las funcionalidades que ofrecen las aplicaciones son muy numerosas y con las nuevas demandas que requieren los clientes de hoy día, se están desarrollando aplicaciones con utilidades profesionales y de ocio, que jamás podíamos haber imaginado, esta sinergia de creatividad está promoviendo nichos de mercado en este campo, con la intención de atender las necesidades de los usuarios y ofrecerles un trato más personalizado. Los nuevos usuarios de smartphone son personas bifuncionales, que manejan la realidad del mundo donde viven y se benefician de la ventana virtual que le ofrece el dispositivo móvil. Este dispositivo ha cambiado el comportamiento y la forma de desenvolverse de las personas en el entorno donde se encuentra, ha generado en nuestras vidas los parámetros de Humano-Movilidad-Aplicada (HuMobAp), es decir, los humanos se mueven en función de las necesidades e incentivos que las aplicaciones les reportan (Florido-Benítez y del Alcázar, 2014).

Como expone Wang, Xiang y Fesenmaier (2014) la conexión constante con las fuentes de información en línea permite a los turistas ser más flexible mediante el aprovechamiento de planificación en línea, por lo tanto, los turistas ajustan sus actividades y planes en consecuencia cuando los incidentes no satisfactorios se llevan a cabo y en última instancia se vuelven más confiados en us viajes. Asimismo, nos alineamos con la mayoría de los autores de esta revisión bibliográfica aportada en esta investigación, ya que ellos, defienden que cuando estamos en un proceso de decisión de compra, información o selección de productos y servicios turísticos, estamos condicionados por nuestra cultura, educación, sexo, edad, ingresos, experiencias, clases de servicios y como se ofrecen a través de los canales de distribución esos servicios. Destacar que estamos en un medio interactivo, frente a una pequeña pantalla móvil y con ciertas limitaciones manuales, visuales y sobre todo no tenemos indicadores de calidad del producto que vamos a seleccionar. Al final, todas estas variables tienen que configurarse en los smartphones con la mayor claridad y seguridad posible, pues ello, condicionará la decisión de su compra y una futura experiencia satisfactoria.

\section{CONCLUSIONES}

Los gestores de la promoción de los destinos y las empresas mercantiles que participan en la actividad turística, tienen que ser conscientes de la importancia de las características demográficas y situacionales en la toma de decisión de los productos y servicios ofrecidos a través de su página web o app del destino turístico. Como se expone en dicho trabajo, los usuarios pasan más tiempos con los dispositivos móviles, son consumidores que demandan mayor inmediatez de información, productos y servicios. La ubicuidad es un factor elemental en el devenir de la toma de decisión de un consumidor, por ello, la interoperabilidad entre los agentes implicados en el ofrecimiento y compra de los productos y servicios a través de la herramienta mobile marketing tiene que ser impecable.

La atomización del sector turístico, ha provocado que tantos las instituciones públicas y empresas privadas, personalicen la oferta en función de las características demográficas: 
género, estado civil, ingresos, ocupación, nacionalidad, etc. y las características situacionales: experiencia, clase de servicio, transversalidad interaccional digital, imagen, seguridad, conexión a internet, etc. Todos estos factores están interconectados en una red digital, en la cual en función de las necesidades y preferencias del consumidor condicionarán la evaluación de satisfacción que el cliente ha obtenido con el producto consumido. Por todo ello, no podemos tratar a todos los clientes por igual, en la revisión bibliográfica de este trabajo investigación mostramos como las mujeres están más predispuestas a los medios de comunicación y poseen una mayor percepción de satisfacción, actitud y uso habitual de internet móvil que los hombres. Así como tener en cuenta, los factores conductuales: raza, cultura, hábitos de compras, leyes, etc., del país donde vamos a promocionar u ofertar los productos o servicios de nuestros clientes potenciales.

Finalmente, se evidencia en esta investigación que la satisfacción al ser un constructo multidimensional no obtiene los mismos resultados en un entorno físico que interactivo. En el medio interactivo la inversión en seguridad tiene que prevalecer sobre otros factores tecnológicos como la creatividad, imagen, utilidad, compatibilidad, etc., ya que si si no se gestionan bien por parte de las empresas, una mala experiencia se transforma en desventajas para el cliente y al final promedian negativamente en la toma de decisión del turista y la satisfacción global. Se tiene que potenciar las ventajas de la herramienta mobile marketing con la finalidad de proyectar seguridad y buena imagen a través de una pantalla reducida, y para lograrlo, el equipo creativo tendrá que hacer un esfuerzo añadido para crear un mensaje que transmita el concepto que se comunica a través de otros medios, pero adaptados a las características del móvil, especialmente el tamaño de la pantalla y el momento de la recepción.

\section{BIBLIOGRAFÍA}

ALBA, W. y HUTCHINSON, J. (1987): «Dimensions of Consumer Expertise», Journal of Consumer Research, vol.13, $\mathrm{n}^{\mathrm{o}}$ 4, pp. 411-454.

AL-DEBEI, M. y AL-LOZI, E. (2014): «Explaining and predicting the adoption intention of mobile data services: A value-based approach», Computer in Human Behavior, vol. 35, 326-338.

ANDERSON, W., FORNELL, C. y LEHMANN, R. (1994): «Customer satisfaction, market share and profitability: finding from », Journal of Marketing, vol. 58, $\mathrm{n}^{\circ} 3$, pp. 53-66.

ANDERSON, S., KLEIN, L. y WIDENER, S. (2008): «Drivers of Service Satisfaction: Linking customer satisfaction to the service concept and customer characteristics», Journal of Service Research, vol. 10, nº 4, pp. 365-381.

AKROUSH, N., AL-MOHAMMAD, M., ZURIEKAT, I. y ALGUL-LAIL, N. (2011): «An empirical model of customer loyalty in the Jordanian Mobile Telecommunications Market», International Journal of Mobile Communications, vol. 9, n ${ }^{\circ}$ 1, pp. 76-101.

BAÑOS, M. (2007): «La creatividad ¿publicitaria? en el marketing móvil», Creatividad y Sociedad, vol.11, pp. 27-52.

BELLMAN, S., POTTER, R., HASSARD, S., ROBINSON, A. \& VARAN, D. (2011): «The Effectiveness of Branded Mobile Phons Apps», Journal of Interactive Marketing, vol. 25, nº, pp. 191-200. 
BIGNÉ, E. y ANDREU, L. (2005): «Emociones, satisfacción y lealtad de visitantes de museos interactivos», Revista Europea de Dirección y Economía de la Empresa, vol. $14, \mathrm{n}^{\mathrm{o}} 2$, pp. 177-190.

BRYANT, B. y CHA, J. (1996): «Crossing the Threshold», Marketing Research, vol. 8, $\mathrm{n}^{\mathrm{O}} 4$, pp. 20-28.

BUTCHER, K. (2005): «Differential Impact of Social Influence in the Hospitality Encounter», International Journal of Contemporary Hospitality Management, vol.17, $\mathrm{n}^{\circ} 2$, pp. $125-135$.

CASTILLO, F. (2010): «Transversalidad, interdisciplinariedad y aprendizaje, una tríada que emerge de la espacialidad», Revista Educación física y deporte, vol. 29, n 2, pp. 279-286.

CHASE, R. y STEWART, D. (1994): «Make Your Service Fail-Safe» Sloan Management Review, vol. 35, n ${ }^{\circ} 3$, pp. 35-44.

CHOI, J., SEOL, H., LEE, S., CHO, H. y PARK, Y. (2008): «Customer satisfaction factors of mobile commerce in Korea», Internet Research, vol.18, n 3, pp. 313-335.

COHEN, L. y MANION, L. (2002): Métodos de investigación educativa, La Muralla. 97. COOIL, B., TIMOTHY L., KEININGHAM, A. y HSU, M. (2007): «A Longitudinal Analysis of Customer Satisfaction and Share of Wallet: Investigating the Moderating Effect of Customer Characteristics», Journal of Marketing, vol. 7, $\mathrm{n}^{\circ} 1$, pp. 67-83.

COOK, P., GOH, C. y CHUNG, C. (1999): «Service Typologies: A State of the Art Survey», Production and Operations Management, vol. 8, no 3, pp. 318-338.

COSTA, E., BARRAGÁNS, B. y REY, M. (2012): «Which App? A recommender system of applications in markets: Implementation of the service for monitoring users' interaction», Expert Systems with Applications, vol. 39, n 10, pp. 9.367-9.375.

DAHLBERG, T. y ÖÖRNI, A. (2007): «Understanding changes in consumer payment habits do mobile payments and electronic invoices attract consumers?» Proceedings of 40th Hawaii International Conference on System Sciences. Washington, DC: IEEE.

DANAHER, J. (1998): «Customer Heterogeneity in Service Management», Journal of Service Research, vol. 1, n ${ }^{\circ}$, pp. 129-139.

DICKINGER, A., HAGHIRAN, P., MURPHY, J. y SCHARL, A. (2004): «An investigation and conceptual modelo SMS marketing» 38th Hawaii International Conference on System Sciences (HICSS-38).

EAGLY, H., MAKHIJANI, G. y KLONSKY, G. (1992): «Gender and the Evaluation of Leaders: A Meta-Analysis», Psychological Bulletin, vol.111, n 1, pp. 3-22.

FLORIDO-BENÍTEZ, L. y DEL ALCÁZAR, B. (2014): «Analysis of Mobile Marketing in Airports» 7th World Conference for Graduate Research in Tourism, Hospitality and Leisure, Istanbul, Turkey: pp. 409-414.

FLORIDO-BENÍTEZ, L., DEL ALCÁZAR, B. y GONZÁLEZ, E. (2014a): «La implementación de las aplicaciones móviles en los aeropuertos para incrementar los niveles de satisfacción del pasajero», I Simposio Internacional y Marketing Turístico IMAT, Valencia, España.

FLORIDO-BENÍTEZ, L., DEL ALCÁZAR, B. y GONZÁLEZ, E. (2014b): «Ventajas e inconvenientes de la herramienta mobile marketing en los niveles de satisfacción de 
los usuarios de dispositivos móviles», International Conference TMS ALGARVE 2014, Perspectivas Contemporâneas em Tecnologias de Informação e Comunicação e em Estrategia, Algarve, Portugal, pp. 69-83.

FLURRY INSIGHTS (2015): «How Americans spend their time on mobile devices», Disponible en: http://flurrymobile.tumblr.com/post/127638842745/seven-years-intothe-mobile-revolution-content-is

FOLKES, S. (1988): «Recent Attribution Research in Consumer Behavior: A review and new directions», Journal of Consumer Research, vol.14, n 4, pp. 548-565.

GARCÍA, D. (2012): Marketing y Negocios en móviles, Wolters Kluwer, S.A, Valencia.

GAVALAS, D. y KENTERIS, M. (2011): "A web-based pervasive recommendation system for mobile tourist guides», Pers Ubiquit Comput, vol. 15, n 7, pp. 759-770.

GIESE, L. y COTE, A. (2000): «Defining consumer satisfaction», Academy of Marketing Science Review, vol. 1, n ${ }^{\circ}$ 1, pp. 1-34.

GIL, I., RUIZ, M., BERENGUER, G. y CORRALIZA, A. (2012): «Determinantes de la lealtad hacia la tienda: la influencia del capital de marca de la tienda, de la satisfacción y de las características personales», XXIV Congreso Nacional de Marketing. Madrid: ESIC.

GILBERT, W. y WARREN, E. (1995): «Psychographic Constructs and Demographic Segments», Psychology \& Marketing, vol. 12, n 3, pp. 223-337.

HELGESEN, O. (2007): «Drivers of customer satisfaction in business-to-business relationships», British Food Journal, vol. 109, n 10, pp. 819-837.

HERMOSO DE MENDOZA, C. (2004): «Móviles, nuevas vías a la comunicación», IPMARK, vol. 616, pp. 30-34.

HOPKINS, J. y TURNER, J. (2012): Mobile Marketing.Marketing basado en localización, aplicaciones, publicidad optimizada, códigos 2D y otras estrategias para hacer crecer su negocio, John Wiley \& Sons, Inc., Hoboken: New Jersey.

HUI, T., WAN, D. y HO, A. (2007): «Tourists' satisfaction, recommendation and revisiting Singapore», Tourism Management, vol. 28, n 4, pp. 965-975.

IACOBUCCI, D. y OSTROM, A. (1993): «Gender Differences in the Impact of Core and Relational Aspects of Services on the Evaluation of Service Encounters», Journal of Consumer Psychology, vol. 2, $\mathrm{n}^{\circ}$ 3, pp. 257-286.

JANIS, L. y MANN, L. (1977): Decision-making. A psychological analysis of conflict, choice and commitment, Nueva York: The Free Press.

JOHNSON, D. y FORNELL, F. (1991): «A Framework for Comparing Customer Satisfaction across Individuals and Product Categories», Journal of Economic Psychology, vol. 12, n 2, pp. 267-286.

JOHN, D. y COLE, C. (1986): «Age differences in information processing: Understanding deficits in young and elderly consumers», Journal of Consumer Research, vol. 13, $\mathrm{n}^{\mathrm{O}}$ 3, pp. 297-315.

JONES, A. y SUCH, J. (2000): «Transaction-specific satisfaction and overall satisfaction: an empirical análisis», Journal of Service Marketing, vol. 14, n 2, pp. 147-159.

LIÉBANA-CABANILLAS, F., SÁNCHEZ-FERNÁNDEZ, J. y MUÑOZ-LEIVA, F. (2014): «Antecedents of the adoption of the new mobile systems: The moderating effect of age», Computer in Human Behavior, vol. 35, pp. 464-478. 
LIN, H. y WANG, Y. (2006): «An examination of the determinants of customer loyalty in mobile commerce contexts», Information \& Management, vol. 43, n 3, pp. 271-282.

LIM, H. y KUMAR, A. (2008): «Gender and loyalty in the context of mobile services», International Journal of Mobile Communications, vol. 6, nº 6, pp. 714-728.

LUBBE, B. (2007): «The effect of Internet apprehension and website satisfaction on air travellers' adoption of an airline's website», Journal of Air Transport Management, vol. $13, \mathrm{n}^{\mathrm{o}} 2$, pp.75-80.

JINZHU, S. (2014): «Understanding the adoption of mobile innovation in China», Computers in Human Behavior, vol. 38, pp. 339-348.

KAPLAN, M. (2012): «If you love something, let it go mobile: Mobile marketing and mobile social media 4x4», Business Horizons, vol. 55, n 2, pp. 129-139.

KUO, Y., WU, M. y DENG, J. (2009): «The relationships among service quality, perceived value, customer satisfaction, and post-purchase intention in mobile value-added services», Computers in Human Behavior, vol. 25, nº 4, pp. 887-896.

MALLAT, N. (2007): «Exploring consumer adoption of mobile payments a qualitative study», The Journal of Strategic Information Systems, vol. 16, $\mathrm{n}^{\circ}$ 4, pp. 413-432.

MARTÍN DE BERNARDO, C. y PRIEDE, T. (2007): Marketing Móvil: Una nueva herramienta de comunicación, Netbiblo, La Coruña.

MCDOUGALL, G. y LEVESQUE, T. (2000): «Customer Satisfaction with Services: Putting Perceived Value into the Equation», Journal of Services Marketing, vol. 14, $\mathrm{n}^{\mathrm{o}} 5$, pp. 392-405.

MCMILLAN, J. y SCHUMACHER, S. (2005): Investigación educativa: una introducción conceptual, Pearson Addison Wesley.

MITTAL, V. y KAMAKURA, A. (2001): «Satisfaction, Repurchase Intent, and Repurchase Behavior: Investigating the Moderating Effect of Customer Characteristics», Journal of Marketing Research, vol. 38, n 1, pp. 131-142.

MELENDO, L. (2010): Aportaciones del teléfono móvil al marketing. Situación y usos en España en el año 2009. Tesis doctoral. Universidad Europe de Madrid.

MELENDO, L. (2012): «Claves para valorar el marketing móvil», Revista de comunicación Vivat Academia, vol.14, pp. 51-62.

MOBILE MARKETING ASSOCIATION (2009): «Marketing Mobile», Disponible en: http:// www.mmaspain.com/index.htm

MMA (2011): «Libro Blanco de apps», Disponible en: http://www.google.com/url?sa=t\& $\mathrm{rct}=\mathrm{j} \& \mathrm{q}=\& \mathrm{esrc}=\mathrm{s} \&$ source $=$ web $\& \mathrm{~cd}=10 \& \mathrm{sqi}=2 \& \mathrm{ved}=0 \mathrm{CHwQFjAJ} \&$ url $=\mathrm{http} \% 3 \mathrm{~A} \% 2$ F\%2Fmmaspain.com\%2Flibro-blanco-apps\%2FRecursos\%2Flibroblanco.pdf\&ei=kQ TvUOqhBoi1hAeq1oG4Cw\&usg=AFQjCNEgdYZYeGZjIYTZlb4T87G4at5CTA\&si g2=m2q8rB_RoEH-01-w2MFTvQ\&bvm=bv.1357700187,d.d2k

SÁNCHEZ,M. (2014): «Cinco cosas de España que no gustan a los chinos», Disponible en: http://noticias.lainformacion.com/economia-negocios-y-finanzas/turismo-y-tiempolibre/cinco-cosas-de-espana-que-no-gustan-a-los-turistas-chinos_tPLgjs9SfTb6ey2znYQk86/

SMITH, B. y MOSCHIS, P. (1990): «The socialization of approach to the study of the elderly consumer», American Marketing Association, pp. 190-226. 
SPRENG, A., MACKENZIE, B. y OLSHAVSKY, W. (1996): «A reexamination of the determinants of consumer satisfaction», Journal of Marketing, vol. 60, $\mathrm{n}^{\circ}$ 3, pp.15-32.

NORM, R. (2011): «Mobile Marketing: How are consumers reacting?», PhoCusWright Innovation Eddition, pp. 1-8.

OKAZAKI, S. y HIROZE, M. (2009): «Does gender affect media choice in travel information search? On the use of mobile Internet», Tourism Management, vol. 30, $\mathrm{n}^{\circ} 6$, pp. 794-804.

OLIVER, R. (1993): "Cognitive, affective and attribute bases of the satisfaction response», Journal of Consumer Research, vol. 20, n 3, pp. 418-430.

PALOS, J. (1998): Educar para el futuro. Temas transversales, Madrid: Desclée de Brouwer.

RAMÓN, D. (2014): «Cómo cambiar la experiencia del pasajero aéreo con inteligencia empresarial», Hosteltur, vol. 241, pp. 46-47.

REINARTZ, W. y KUMAR, V. (2002): «The Mismanagement of Customer Loyalty», Harvard Business Review, vol.80, pp. 86-94.

SAN MARTÍN, S. y LÓPEZ, B. (2012): «Determinantes personales de la satisfacción del comprador por el teléfono móvil», XXIV Congreso Nacional de Marketing. Madrid: ESIC.

SANZ, S., MARTÍ, J. y RUIZ, C. (2012): «Aplicaciones publicitarias para móvil: conocimiento, actitudes, motivos de uso y valoración por parte de los adolescentes españoles», Pensar la Publicidad. Revista Internacional de Investigaciones Publicitarias, vol. $6, \mathrm{n}^{\circ} 1$, pp. 255-270.

SASSER, W., OLSEN, P. y WYCKOFF, D. (1978): Management of Service Operations, Boston: Allyn \& Bacon.

SCOLARI, C., NAVARRO, H., PARDO, H., GARCÍA, I. y SORIANO, J. (2009): «Comunicación móvil: actores y producción de contenidos en Cataluña», Comunicación y Sociedad, vol. 22, n 2, pp. 159-185.

SHANKAR, V. y BALASUBRAMANIAN, S. (2009): «Mobile Marketing: A Synthesis and Prognosis», Journal of Interactive Marketing, vol. 23, $\mathrm{n}^{\circ}$ 2, pp. 118-129.

SONG, J. (2014): «Understanding the adoption of mobile innovation in China», Computers in Human Behavior, vol. 38, pp. 339-348.

THE APPDATE (2012): «Apps Report September 2012», Disponible en: http://madrid. theappdate.com/informe-apps/

TUSSYADIAH, P. (2012): «A concept of location-based social network marketing», Journal of Travel \& Tourism Marketing, vol. 29, $\mathrm{n}^{\circ} 3$, pp. 205-220.

VANHAMME, J. y SNELDERS, D. (2001): «The role of surprise in satisfaction judgements», Journal of Consumer Satisfaction Dissatisfaction and Complaining Behavior, vol. 14, pp. 27-45.

VLACHOS, P. y VRECHOPOULOS, A. (2007): «Mobile Marketing - Achieving Competitive Advantage through Wireless Technology», Journal of Services Marketing, vol. $21, \mathrm{n}^{\circ} 7$, pp. 539-541.

WANG, D., PARK, S. y FESENMAIER, R. (2012): «The role of smartphones in mediating the Touristic experience», Journal of Travel Research, vol. 51, $\mathrm{n}^{\circ} 4$, pp. 371-387. 
WANG, D., XIANG, Z. y FESENMAIER, R. (2014): «Adapting to the mobile world: A model of smartphone use», Annals of Tourism Research, vol. 48, pp. 11-26.

WANGENHEIM, F. (2003): «Situational characteristics as moderators of the satisfaccionloyalty link: an investigation in a business-to.business context», Journal of Consumer Satisfaction Dissastifaction and Complaining Behavior, vol.16, pp. 145-156.

WOODRUFF, B., CADOTTE, E. y JENKINS, R. (1983): «Modeling Consumer Satisfaction Processes Using Experience-Based Norms», Journal of Marketing Research, vol. 20, nº 3, pp. 296-304.

WOODRUFF, B. y GARDIAL, F. (1996): Know Your customer: New Approaches to Understanding Customer Value and Satisfaction, Blackwell Publishers.

YANG, Z. y PETERSON, R. (2004): «Customer perceived value, satisfaction, and royalty: the role of switching costs», Psychology and Marketing, vol. 21, n 10, pp. 779-822.

YU, T. y DEAN, A. (2001): «The contribution of emotional satisfaction to consumer loyalty», International Journal of Service Industry Management, vol. 12, $\mathrm{n}^{\circ} 3$, pp. 234-250.

ZEITHAML, V. (1985): «The New Demographics and Market Fragmentation», Journal of Marketing, vol. 49, $\mathrm{n}^{\circ}$ 3, pp. 64-75.

ZEITHAML, V. y BITNER, M. (2002): Services Marketing: Integrating Customer Focus Across the Firm, 3rd ed. New York: McGraw Hill/ Irwin. 
